\title{
Optic Disc Detection Using Ant Colony Optimization
}

\author{
Marcy A. Dias and Fernando C. Monteiro \\ Polytechnic Institute of Bragança, Bragança, Portugal
}

\begin{abstract}
The retinal fundus images are used in the treatment and diagnosis of several eye diseases, such as diabetic retinopathy and glaucoma. This paper proposes a new method to detect the optic disc (OD) automatically, due to the fact that the knowledge of the OD location is essential to the automatic analysis of retinal images. Ant Colony Optimization (ACO) is an optimization algorithm inspired by the foraging behaviour of some ant species that has been applied in image processing for edge detection. Recently, the ACO was used in fundus images to detect edges, and therefore, to segment the OD and other anatomical retinal structures. We present an algorithm for the detection of OD in the retina which takes advantage of the Gabor wavelet transform, entropy and ACO algorithm. Forty images of the retina from DRIVE database were used to evaluate the performance of our method.
\end{abstract}

Keywords: Ant Colony Optimization, Gabor Wavelet Transform, Entropy, Optic Disc Detection and Retinal Images PACS: $87.57 . \mathrm{N}-$

\section{INTRODUCTION}

The segmentation of the OD, in addition of being important in several pre-processing algorithms developed for the automatic extraction of anatomical structures and to detect damage to the retina, is also an indicator of different eye diseases, such as glaucoma which is one of the most common causes of blindness [1]. These images must be accurately segmented to extract sensitive objects in the retina like the blood vessels tree, the OD, the macula and the region between the OD and the macula.

The OD is the brightest area within a retinal image and is the entrance and exit point for retinal blood vessels and, also, the point from where the retinal fibers flow. Furthermore, in retinal images the OD detection is a concern to locate all kinds of anatomical features in the retina.

Several OD detection methods make use of anatomical structures among the OD, macula and retinal blood vessels [2]. These methods are based on the assumption that all major retinal blood vessels radiate from the OD, which make they more reliable under the presence of retinal lesions or/and imaging artefacts. Even though, the extraction of either retinal blood vessels or the macula is often a nontrivial task by itself.

The ACO is an optimization algorithm inspired by the foraging behaviour of some ant species that has been applied in image processing for edge detection [3]. For ants the brightest areas within the image correspond to food supply zones, so they mark these areas as important zones which become a good indicator to the optic disc location.

Our method starts with the blood vessels segmentation followed by the entropy filtering and then it was applied the ant colony optimization for the detection of the OD. These approaches are then joint to obtain a set of candidates for the OD location.

\section{MATERIALS AND METHODS}

The algorithm was tested in forty images from DRIVE database (seven of which present pathology), compressed in JPEG format of size $565 \times 584$ pixels, eight bits per colour channel, obtained through a diabetic retinopathy screening program. These images area were acquired using a Canon CR5 nonmydriatic 3CCD camera at $45^{\circ}$ field of view.

\section{Blood Vessels Segmentation}

The most important step in many different applications of image processing is to detect the blood vessels in the retina. In our work, we decided to choose the method presented in [4] due to the fact that they achieved an high performance in blood vessels segmentation $(\approx 96 \%)$. In this method each image pixel is represented by a feature

Numerical Analysis and Applied Mathematics ICNAAM 2012

AIP Conf. Proc. 1479, 798-801 (2012); doi: 10.1063/1.4756258

(C) 2012 American Institute of Physics 978-0-7354-1091-6/\$30.00 
vector including measurements at different scales taken from the two-dimensional (2D) Gabor wavelet transform. The resulting feature space is used to classify each image pixel as either a vessel or nonvessel pixel. This is done using a Bayesian classifier which is described as Gaussian mixtures, yielding a fast classification, while being able to model complex decision surfaces [4].

The 2D Gabor wavelet is defined as:

$$
\psi_{G}(\mathbf{x})=\exp \left(j \mathbf{k}_{\mathbf{0}} \mathbf{x}\right) \exp \left(-0.5|\mathbf{A x}|^{2}\right)
$$

where $\mathbf{k}_{\mathbf{0}}$ is a vector that defines the frequency of the complex exponential and $A=\operatorname{diag}\left[\frac{1}{\sqrt{\varepsilon}}, 1\right]$ is a $2 \times 2$ diagonal matrix that defines the anisotropy of the filter, i.e., its elongation in any desired direction. This wavelet is computed for spanning from 0 up to 170 degrees at steps of 10 degrees. The maximum value of the wavelet transform over all angles for various scales are then taken as pixel features [4]. In our approach, the elongation parameter was set to $\varepsilon=4$ and $\mathbf{k}_{\mathbf{0}}=3$ (see Fig. 1(b)).

\section{Entropy}

The entropy is typically used to detect the exudates in retinal images. In our method, we used entropy to detect optic disc due to the fact that it is easily distinguished from the rest of the retina through its smooth texture [5]. To discover which regions of the image are smooth or textured, for each point $x$ we obtained a probability mass function $P_{I_{x}}:\{0,1, \ldots, 255\} \mapsto[0 . .1]$ for the intensities $I_{x}$ of the pixels in a local region around $x$ and then we compute the entropy:

$$
H\left(I_{x}\right)=-\sum_{i=0}^{255} P_{I_{x}}(i) \cdot \log P_{I_{x}}(i),
$$

This entropy measure is higher when the region around a pixel is complex and low when it is smooth. After applying the entropy filter we implemented an adaptive threshold to separate the complex regions from the smoother ones (see Fig. 1(c)) [5].

\section{Ant Colony Optimization}

Recently, the ACO was used in fundus images to detect edges, and therefore, to segment the OD and other anatomical retinal structures $[3,6]$.

This method uses a number of ants on the image driven by the local variation of the image intensity values. This variation establishes a pheromone matrix that represents the edge information at each image pixel location. The process starts with an initialization stage, and then runs for $\mathrm{N}$ iterations to construct the pheromone matrix by iteratively performing both the construction and the update processes.

The algorithm contains two crucial issues that have to be considered in the ACO process: the establishment of the probabilistic transition matrix $p^{(n)}$ and the pheromone matrix $\tau^{(n)}$ update. For the first one, we used a probabilistic action rule determined by [7]:

$$
p_{(l, m),(i, j)}^{n}=\frac{\left(\tau_{i, j}^{(n-1)}\right)^{\alpha}\left(\eta_{i, j}\right)^{\beta}}{\sum_{(i, j) \in \Omega_{(l, m)}}\left(\tau_{i, j}^{(n-1)}\right)^{\alpha}\left(\eta_{i, j}\right)^{\beta}}, i f(i, j) \in \Omega_{(l, m)}
$$

which indicates the probability that at the n-th construction step of ACO, the k-th ant moves from the node $(l, m)$ to the node $(i, j)$. In Equation $(1), \tau_{i, j}^{(n-1)}$ is the pheromone information value of the arc linking the two nodes under consideration, $\Omega_{(l, m)}$ is the set of feasible components like edges $(l, m),(i, j)$ where $(i, j)$ is a nod not yet visited by the ant $k, \alpha$ and $\beta$ are,respectively, constants that represent the influence of the pheromone and the heuristic information and $\eta_{i, j}$ represents the heuristic information for going from node $(l, m)$ to the node $(i, j)$, fixed to be the same for each construction step.

This parameter was found by the local statistics at the pixel position $(i, j)$ as:

$$
\eta_{i, j}=\frac{1}{Z} V_{c}\left(I_{i, j}\right)
$$




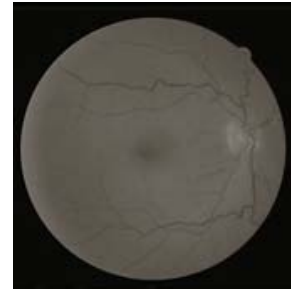

(a)

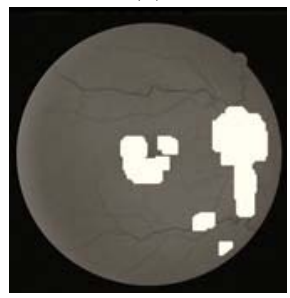

(d)

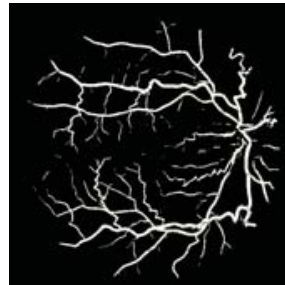

(b)

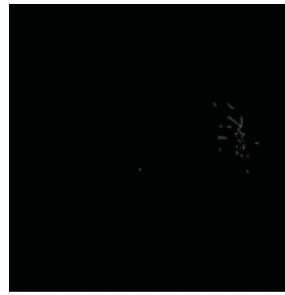

(e)

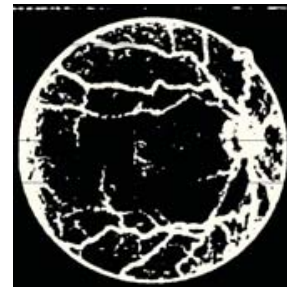

(c)

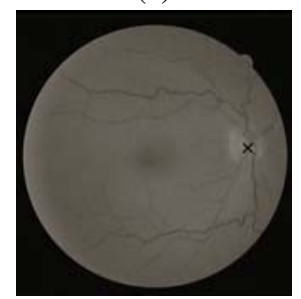

(f)

FIGURE 1. Proposed method applied to one of the fundus image. (a) original gray-level image (b) blood vessel segmentation (c) entropy filter (d) ACO result within original image (e) final OD-center candidates (f) detection of OD

where $Z=\sum_{i=1: M_{1}} \sum_{j=1: M_{2}} V_{c}\left(I_{i, j}\right)$ is a normalization factor, $I_{i, j}$ is the intensity value of the pixel at the position $(i, j)$ of the image $I$, and $V_{c}\left(I_{i, j}\right)$ is the function of a local group of pixels $c$ (called the clique).

In order to establish the optimal function, four different approaches are considered as in [6]. The highest performance was obtained with the expression in Equation (2) where the parameter $\lambda$ define the function shape.

$$
f(x)=\left\{\begin{array}{c}
\frac{\pi x \sin \left(\frac{\pi x}{\lambda}\right)}{\lambda}, \quad 0 \leq x \leq \lambda \\
0, \quad \text { otherwise }
\end{array}\right.
$$

As far as the pheromone matrix is considered it needs to be updated twice during the ACO process. The first update occurs after the movement of each ant within each construction step. This update is performed using Equation (3):

$$
\tau_{i, j}^{(n-1)}=\left\{\begin{array}{c}
(1-\rho) \cdot \tau_{i, j}^{(n-1)}+\rho \cdot \Delta_{i, j}^{(k)}, \quad \text { if }(i, j) \text { is visited by the current } \mathrm{k}-\mathrm{th} \text { ant } \\
\tau_{i, j}^{(n-1)}, \quad \text { otherwise }
\end{array}\right.
$$

where $\rho$ is the evaporation rate, $\Delta_{i, j}^{(k)}$ is determined by the heuristic matrix, which is $\Delta_{i, j}^{(k)}=\eta_{i, j}$. The second update occurs after the movement of all $K$ ants within each construction step and the matrix is updated according to:

$$
\tau^{(n)}=(1-\varphi) \cdot \tau^{(n-1)}+\varphi \cdot \tau^{0}
$$

where $\varphi \in(0,1]$ is the pheromone decay coefficient.

Another parameter that had to be established was the permissible range of ants movement $\Omega_{(l, m)}$. In this paper, we used the 8-connectivity proposed by [6]. The parameter values of the ACO algorithm were: $K=\left\lfloor\sqrt{M_{1} x M_{2}}\right\rfloor$, $\tau_{\text {initial-value }}=0.0001, \alpha=1, \beta=0.1, \lambda=10, \rho=0.1, L_{\text {steps }}=40, \varphi=0.05$ and $N_{\text {construction-steps }}=3$ (see Fig. $1(\mathrm{~d})$ ). Figure 1(e) is the overlapping of the ACO image with the two images above.

\section{RESULTS AND DISCUSSION}

The proposed method achieved a success rate of $100 \%$ over the DRIVE database. The estimated centre is considered correct if it was positioned within 60 pixels of the manually identified centre, as proposed in [2, 8, 9]. Figure 2 shows the best and the worst four results for the OD detection. The average distance reached between the estimated OD centre and the manually identified centre was 29.9 pixels, the minimum distance was 9.9 and the maximum was 49.2 pixels. 

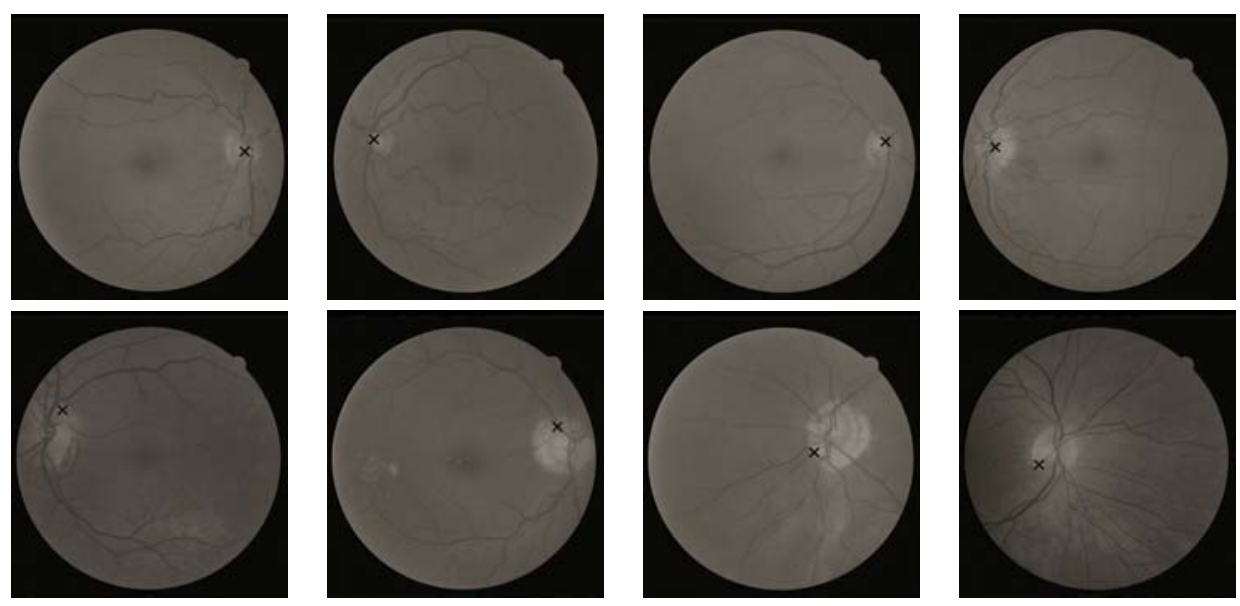

FIGURE 2. OD detection examples (black mark represents the estimated OD centre). The first row shows the best four results. The second row shows the worst four results.

\section{CONCLUSIONS}

In this paper, a simple and computationally efficient algorithm for automatic OD detection was presented. The proposed method takes advantage of the powerful techniques such as the Gabor wavelet transform, entropy and ACO algorithm. The performance of this method was evaluated using the forty images from DRIVE database. Good results have been achieved and the method did not fail in any image.

\section{REFERENCES}

1. J. Xu, P. Chew, IEEE Transactions in Biomedical Engineering 54, 473-482 (2007).

2. A. Youssif, A. Ghalwash, A. Ghoneim, IEEE Transactions on Medical Imaging 27, 11-18 (2008).

3. C. Pereira, L. Gonçalves, M. Ferreira, "Detection of the Optic Disc in Retinal Images by Means of Ant Colony Optimization Algorithm," in The 2011 International Conference on Image Processing, Computer Vision, and Pattern Recognition, Las Vegas, United States of America, 2011, vol. 1.

4. J. Soares, J. Leandro, R. Cesar, H. Jenilek, M. Cree, IEEE Transactions on Medical Imaging 25, 1214-1222 (2006).

5. L. Kovacs, R. Qureshi, B. Nagy, B. Harangi A. Hajdu, "Graph Based Detection of Optic Disc and Fovea in Retinal Images," in 4th International Workshop on Soft Computing Applications, Arad, Romania, 2010, vol. 10, pp. 143-148.

6. J. Tian, W. Yu, S. Xie, “An Ant Colony Optimization Algorithm for Image Edge Detection," in Proceedings of IEEE Congress on Evolutionary Computation, 2008, pp. 751-756.

7. M. Dorigo, M. Birattari, T. Stutzle, IEEE Computational Intelligence Magazine 1, 28-39 (2006).

8. M. Forachia, E. Grisan, A. Ruggeri, IEEE Transactions on Medical Imaging 23, 1189-1195 (2004).

9. A. Hoover, V. Kouznetsova, M. Goldbaum, IEEE Transactions on Medical Imaging 19, 203-210 (2000). 